\title{
Real-Time Classification Technique for Early Detection and Prevention of Myocardial Infarction on Wearable Devices
}

\author{
Dionisije Sopic ${ }^{1}$, Amin Aminifar ${ }^{2}$, Amir Aminifar ${ }^{1}$, David Atienza ${ }^{1}$ \\ ${ }^{1}$ Embedded Systems Lab. (ESL), EPFL, Switzerland, ${ }^{2}$ Khajeh Nasir Toosi University, Iran \\ \{dionisije.sopic, amir.aminifar, david.atienza\}@epfl.ch, \{amin.aminifar\}@email.kntu.ac.ir
}

\begin{abstract}
Continuous monitoring of patients suffering from cardiovascular diseases and, in particular, myocardial infarction (MI) places a considerable burden on health-care systems and government budgets. The rise of wearable devices alleviates this burden, allowing for long-term patient monitoring in ambulatory settings. One of the major challenges in this area is to design ultralow energy wearable devices for long-term monitoring of patients' vital signs. In this work, we present a real-time event-driven classification technique, based on support vector machines (SVM) and statistical outlier detection. The main goal of this technique is to maintain a high classification accuracy while reducing the complexity of the classification algorithm. This technique leads to a reduction in energy consumption and thus battery lifetime extension. We validate our approach on a well-established and complete myocardial infarction (MI) database (Physiobank, PTB Diagnostic ECG database [1]). Our experimental evaluation demonstrates that our real-time classification scheme outperforms the existing approaches in terms of energy consumption and battery lifetime by a factor of 3 , while maintaining the classification accuracy at a medically-acceptable level of $\mathbf{9 0 \%}$.
\end{abstract}

\section{INTRODUCTION AND RELATED WORK}

Cardiovascular diseases and, in particular myocardial infarction (MI), represent one of the leading causes of death nowadays. MI, also commonly known as heart attack, is a worldwide lifethreatening condition, affecting more than 700,000 people only in the USA [2]. The annual costs of US hospitalizations due to MI in 2010 were estimated at over 45 billion US dollars [3].

MI occurs when one of the coronary arteries that supply the oxygenated blood to the heart muscle becomes blocked. This situation occurs due to a build-up of fatty deposits (plaques) that gradually form in one of these arteries. Upon rupture, these plaques release thrombogenic contents that trigger the blood clot to form. The blood clot can completely block an artery resulting in myocardial ischaemia, a diminished blood supply to the part of the heart that was getting supplied by the blocked artery. Without oxygen, muscle cells of this part of the heart begin dying, resulting in a heart attack. The histological cell death begins in as little as 20 minutes [4], [5]. Once MI begins, the goal is to get a person into treatment in less than 90 minutes. If the time between hospital arrival and performance of the treatment, the so-called door-to-balloon time, is longer than 2 hours, the rate of mortality increases significantly by $41-62 \%$ [6]. Moreover, the complete necrosis of myocardial cells at risk typically requires 2 to 4 hours [4]. Thus, prolongation of the period of ischaemia without treatment will have irreversible consequences, i.e., death of the affected cells.

In addition to the previously discussed risks of MI, patients who have already had a heart attack are at increased risk of recurrent infarctions with an annual death rate that is six times higher than in people of the same age who have not had a heart attack [7]. Hence, adequate care should be provided to these patients. Long-term monitoring of these patients has been usually performed in hospitals by bulky medically-approved devices. The major challenges of these devices are their high energy consumption and lack of portability, which makes them inconvenient to be used for ambulatory and home-based monitoring.

The need for regular check-ups of these patients can be alleviated through means of wearable devices that can be used autonomously by patients. These devices provide the possibility of replacing the functionality of hospital equipment with new portable low-cost devices that offer mobility, and long-term patient monitoring on a daily basis. This allows clinicians to detect early symptoms of potential cardiac irregularities and prevent further patient's state deterioration. Subsequently, based on these parameters, care can be provided to patients in the ambulatory settings, which reduces the hospitalization rate and costs. Recent advances in machine learning enable the estimation of these parameters from physiological signals.

Existing machine learning algorithms for early detection and prevention of MI that achieve high classification accuracy are too complex to be implemented on wearable devices for realtime monitoring (K-nearest neighbours, see [8]). Furthermore, these algorithms have to meet stringent energy constraints to be used for long-term patient monitoring. Therefore, the classification algorithms running on a battery-powered wearable device should be highly optimized, which promotes the need for a paradigm shift in the classifier design. The event-driven computing paradigm presents a promising solution to cope with the computational complexity of learning algorithms and, in turn, to substantially reduce the energy consumption.

The need to reduce the energy consumption of wearable devices has given rise to a plethora of studies. In [9], a real-time classification scheme for automatic detection of abnormal heartbeats targeting embedded and resource-constrained Wireless Body Sensor Nodes (WBSNs) has been proposed. This scheme also incorporates an advanced digital signal processing block that is activated just when abnormal beats are detected, which considerably decreases the computational requirements and the energy consumption. First, and from a medical reliability point of view, the authors do not investigate the confidence interval of obtained results, which is an important parameter in medical applications. Secondly, and from an energy-efficiency point of view, their work focuses on a context where pathological heartbeats occur less frequently than normal ones. Therefore, in case of many pathological heartbeats happening one after another, the advanced digital signal processing block will be successively invoked, which will increase the computational complexity and energy consumption. In [8], the authors report a high accuracy in classifying normal and MI ECG beats from 47 features, which is not an appropriate classification scheme for wearable devices due to their stringent energy budgets, as we show in Section III.

In this work we target early detection and prevention of myocardial infarction through means of ultra-low energy wearable devices. We propose a hierarchical real-time event-driven classification technique that reduces the energy consumption, 
while still maintaining the classification accuracy at a medicallyacceptable level of $90 \%$, based on the internal discussion with medical experts. The main contributions of this paper are:

1) Real-time early detection and prevention of myocardial infarction using an event-driven classification technique that uses a two-level classifier, in which the first-level classifier is computationally efficient and cannot provide a medically-acceptable accuracy, whereas the second-level can provide it, but it is computationally more complex.

2) Validation on myocardial infarction (MI) database (Physiobank - PTB Diagnostic ECG database [1])

3) Porting of our classification technique on a wearable platform and evaluation of energy consumption and battery lifetime.

\section{REAL-TIME EVENT-DRIVEN TECHNIQUE}

In this section, we propose a real-time event-driven learning technique, based on support vector machines (SVM) [10] and statistical outlier removal [11]. The goal is to maintain high accuracy while reducing the expected complexity. Reducing computational complexity, in turn, results in a longer battery lifetime, which is an important factor for wearable devices. Therefore, we adopt a real-time event-driven computing scheme for early detection and prevention of MI. In an event-driven computing paradigm it is the occurrence of an event rather than the passing of time, which decides when a particular action should be taken [12]. The event-driven schemes perform the most computationally expensive processing only when it is necessary.

The overall flow of our approach is shown in Fig. 1. Our proposed classification technique has 2 main phases: the offline phase that is discussed in Section II-A, and the online phase discussed in Section II-B. The offline phase consists of 2 stages: the training phase and the phase in which we fit distributions to misclassified training examples.

\section{A. Offline phase of our real-time event-driven classifier}

An event-driven classification scheme relies on the fact that most of the time we can make confident decisions based on only a few features and only rarely we need to compute all the features. Therefore, the basic idea here is to use a two-level classifier. The first-level classifier only considers a limited number of features and, therefore, is computationally efficient, but cannot provide medically-acceptable results with $90 \%$ accuracy. On the contrary, the second-level classifier can provide a medically-acceptable accuracy, but is computationally complex. In our real-time event-driven classification technique, the second-level classifier is activated only if the first-level classifier is unable to classify the sample data with the required confidence.

We make two main observations with respect to our realtime event-driven classification scheme: (1) The overall classification complexity is mainly determined by the complexity of the first-level classifier, since the majority of the data samples can be classified based on the first-level classifier relying on a limited number of features. (2) The overall classification accuracy is similar to that of the second-level classifier since when the first-level classifier cannot make confident decisions, the second-level classification is invoked.

Our real-time event-driven classifier incorporates two softmargin SVMs, and the dimensionality of features is different. In the offline learning phase in Fig. 1, we use the matrix of features $\boldsymbol{X}$ and the vector of labels $\boldsymbol{y}$ to obtain the classifier

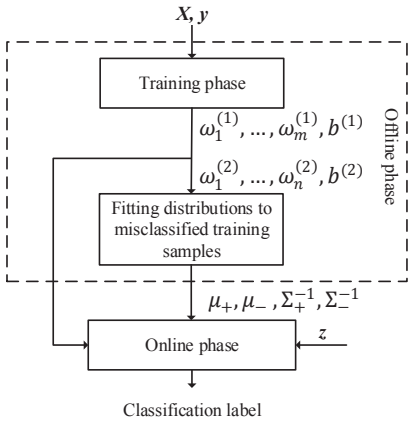

Fig. 1: Flowchart of our two-level classification technique

parameters $\omega_{1}^{(1)}, \ldots, \omega_{m}^{(1)}, b^{(1)}$ for the first-level classifier, and $\omega_{1}^{(2)}, \ldots, \omega_{n}^{(2)}, b^{(2)}$ for the second-level one. The next step is to find the misclassified samples for both classes. These samples are represented by normal distributions with parameters $\left(\boldsymbol{\mu}_{+}, \boldsymbol{\Sigma}_{+}\right)$and $\left(\boldsymbol{\mu}_{-}, \boldsymbol{\Sigma}_{-}\right)$. Parameters $\left(\boldsymbol{\mu}_{+}, \boldsymbol{\Sigma}_{+}\right),\left(\boldsymbol{\mu}_{-}, \boldsymbol{\Sigma}_{-}\right)$ represent the mean vector and the covariance matrix of misclassified samples that correspond to label $y=1, y=-1$, respectively. As shown in [11], given an n-dimensional feature vector $z_{1}$, with the mean vector $\boldsymbol{\mu}$ and covariance matrix $\boldsymbol{\Sigma}$, the scalar random variable $U\left(\boldsymbol{z}_{1}\right)$ that is defined as:

$$
U\left(z_{1}\right)=\left(z_{1}-\boldsymbol{\mu}\right)^{T} \boldsymbol{\Sigma}^{-1}\left(z_{1}-\boldsymbol{\mu}\right),
$$

follows a $\chi^{2}$ distribution with $n$ degrees of freedom, where $n$ is the size of feature vector $z_{1}$ [11]. This metric will be used in the online phase of our technique to determine if it is required to invoke the second-level classifier. Based on the selected confidence level and the number of features in the first-level classifier, we calculate the critical value $\chi_{c}^{2}$. This critical value determines the probability of calling the second-level classifier.

\section{B. Online phase of our real-time event-driven classifier}

The overall flow of the online phase of our proposed two-level classifier is shown in Fig. 2. When classifying new examples, depending on the required confidence level, either the first-level classifier with a reduced set of features, or the secondlevel classifier that uses the entire set of available features will be invoked. Given a feature vector $z$ with features $z_{i}$ for $i=1, \ldots, n$, we first calculate the output of the first-level classifier. This is done simply by evaluating the sign function:

$$
\operatorname{sign}\left(\sum_{i=1}^{m} \omega_{i}^{(1)} z_{i}+b^{(1)}\right) \text {. }
$$

Note that the first-level classifier only uses the first $m$ features. Based on the result that we obtain from Eq. (1), we calculate $U(\boldsymbol{z})$ as follows:

$$
U(z)= \begin{cases}\left(z-\mu_{+}\right)^{T} \Sigma_{+}^{-1}\left(z-\mu_{+}\right), & \text {if } \operatorname{sign}(\mathrm{y})<0 \\ \left(z-\mu_{-}\right)^{T} \Sigma_{-}^{-1}\left(z-\mu_{-}\right), & \text {if } \operatorname{sign}(y)>0\end{cases}
$$

The classifier that is going to be used for classification depends on the value of $\chi_{c}^{2}$ calculated in the offline phase and $U(\boldsymbol{z})$

$$
\text { Classifier }= \begin{cases}\text { First-level classifier, } & \text { if } U(\boldsymbol{z}) \geq \chi_{c}^{2} \\ \text { Second-level classifier, } & \text { otherwise. }\end{cases}
$$




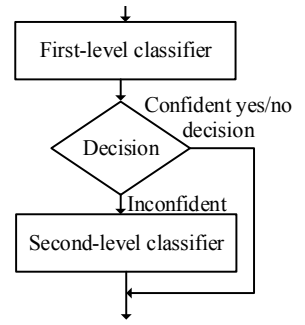

Fig. 2: Flowchart of online phase of our two-level classifier

If $U(\boldsymbol{z})<\chi_{c}^{2}$, the output of the second-level classifier is calculated by evaluating the following function:

$$
\operatorname{sign}\left(\sum_{i=1}^{n} \omega_{i}^{(2)} z_{i}+b^{(2)}\right),
$$

where $n$ is the number of all available features.

\section{Complexity analysis}

The expected complexity (the mathematical expectation denoted by $\mathbb{E}(\cdot)$ ) of our approach is computed as follows:

$$
\mathbb{E}(c)=p c_{1}+(1-p) c_{2} .
$$

where $c_{1}$ is the complexity of the first-level classifier and $U(z)$, and $c_{2}$ is the complexity of computing all the features. The parameter $p$ is defined as the probability that the firstlevel classifier will be sufficient. This probability $p$ depends on the value $\chi_{c}^{2}$, as the first-level classifier will be sufficient for those testing samples for which $U(z) \geq \chi_{c}^{2}$. Namely, an increase in the value of $\chi_{c}^{2}$ leads to an increase in classification accuracy, as the second-level classifier is then invoked more frequently. However, at some point there is no improvement in the classification accuracy, as the second-level classifier is always invoked. On the other hand, this situation also increases the computational complexity. In addition, as we show in Section III, the energy consumption increases as well, which reduces the battery lifetime of wearable devices.

\section{EXPERIMENTAL SETUP}

In this section, we use the ECG signals from Physiobank (PTB Diagnostic ECG database) open access database [1] for validating our technique. Signals from 52 healthy subjects and 52 patients who already had an MI are used for classifying healthy and MI electrocardiogram (ECG) beats.

\section{A. Preprocessing}

As a first step, we extensively filter the ECG signals to remove the noise. Namely, the morphological filtering [13] is used for ECG baseline wander removal. A 32ndorder zero-phase FIR band-pass filter with cut-off frequencies $f_{1}=0.05 \mathrm{~Hz}$ and $f_{2}=40 \mathrm{~Hz}$ is applied for high-frequency noise and artefact removal. The R-peaks of the ECG signals are detected using Pan-Tompkin's algorithm [14]. Similar to [8], after detecting the R-peak, 250 samples to the left and 400 samples to the right of R-peak are used for segmenting each ECG beat. Each of these ECG beats is further decomposed using a discrete wavelet transform (DWT) down to level four, resulting in eight coefficients. DWT was performed using Daubechies 6 (db6) wavelet basis function. We use the same set of features extracted from signals coming from Lead 11 as in [8]. Finally, the forward feature selection procedure [15] is used to find the most informative ones.

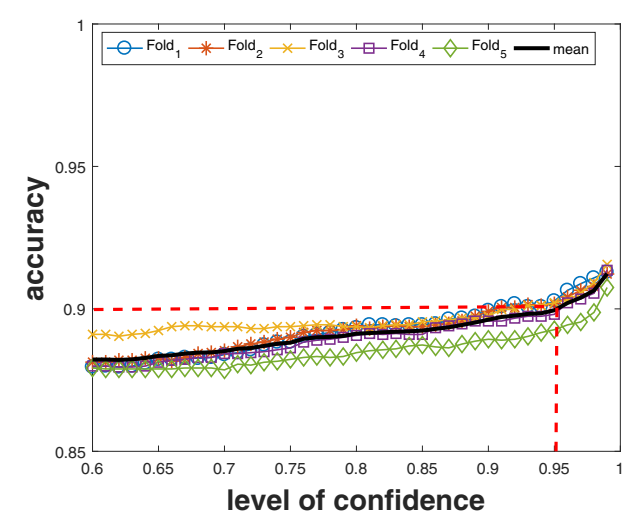

Fig. 3: Accuracy of our technique. Each curve in this figure shows the accuracy obtained on the testing fold, while the classifier has been trained on the remaining four folds. The figure legend indicates the fold used for testing the model.

\section{B. Target platform}

We use the SmartCardia INYU device [16], which is a wearable device used for health monitoring, to evaluate our technique. This device acquires user's ECG signal from an ECG sensor with the sampling frequency that ranges from $125 \mathrm{~Hz}$ up to $16 \mathrm{KHz}$, and with up to 16 -bit resolution. This SmartCardia device has a standard $710 \mathrm{mAh}$ battery. The entire processing is done on an ultra-low power 32-bit microcontroller STM32L151 [17] with an ARM $^{\circledR}{ }^{\circledR}$ Cortex $^{\circledR}$ M3 that can operate at a maximum frequency of $32 \mathrm{MHz}$. This device has $48 \mathrm{~KB}$ RAM, $384 \mathrm{~KB}$ Flash and several analog peripherals including a 12-bit ADC. More details about this device could be found in [18].

\section{Accuracy and computational complexity}

In this subsection, we calculate the classification accuracy along with the computational complexity of our classification technique. As our dataset is balanced, the classification accuracy is a valid metric for classifier performance evaluation, and it is defined as follows:

$$
\text { Accuracy }=\frac{t p+t n}{t p+t n+f p+f n},
$$

where $t p, t n, f p, f n$ represent the number of true positive, true negative, false positive, and false negative, respectively. It is derived from a five-fold cross-validation process, with samples of different classes randomly divided across folds. Out of the five folds, a single fold is retained for testing the model, and the remaining four are used as training data. For instance, Fold 1 curve in Fig. 3 corresponds to testing based on Fold 1 and training using the remaining four folds. We consider $n=47$ features to be consistent with previous studies in [8]. The number of features used in the first-level classifier is $m=5$.

Fig. 3 shows the overall classification accuracy of our proposed classification scheme (vertical axis) versus the required confidence level $\sigma$ (horizontal axis). From Fig. 3, we see that an increase in confidence level $\sigma$ leads to an increase in classification accuracy. This holds true, as an increase of confidence level results in invoking the second-level classifier more frequently. Based on Eq. (2), we calculate the expected computational complexity of our technique versus the required confidence level. As shown in Fig. 4, the second-level classifier is not invoked all the time. In fact, even if we increase the 


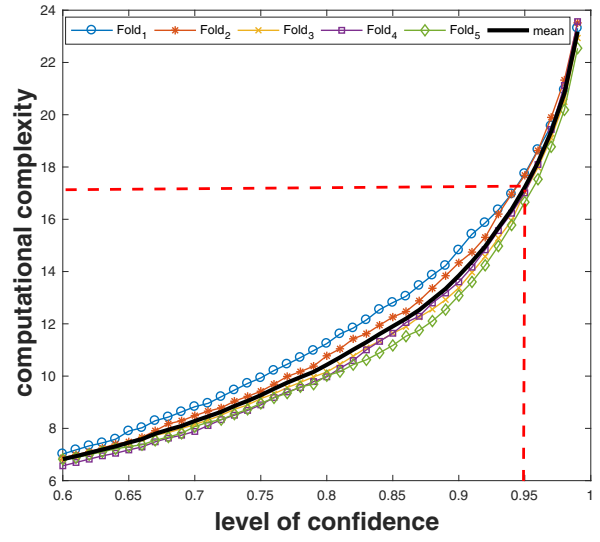

Fig. 4: Estimated computational complexity of our real-time event-driven technique. Each curve in this figure shows the computational complexity obtained on the testing fold, while the classifier has been trained on the remaining four folds. The figure legend indicates the testing fold.

confidence level to $\sigma=0.99$, the expected complexity of our approach is less than 24 in terms of the number of features, which is almost half of the proposed approach in [8].

For higher confidence level, higher computational complexity is expected, as shown in Fig. 4. In this figure, we can deduce that our approach reduces the classification complexity by almost a factor of 3, as the maximal number of used features is 17.22 in comparison with 47 available features. After adapting the algorithm proposed in [8] to SVM algorithm, it is possible to reach an accuracy of $95 \%$. As our technique still provides a medically-acceptable accuracy of $90 \%$, this loss of accuracy results in the computational complexity that is almost 3 times lower in comparison with the computational complexity of the proposed approach in [8].

\section{Energy consumption}

We compare the energy consumption of our real-time event-driven technique against the proposed approach in [8] on an actual hardware platform, the SmartCardia device. The comparison with previous work is outlined in Table I. For a standard $710 \mathrm{mAh}$ battery, assuming that the processing is done once per minute, the method proposed in [8] runs for 120.64 hours on a single battery charge. Our proposed classification technique, however, reaches 394.58 hours, thus allowing for more that 16 days of operation. Therefore, our event-driven classification technique extends the battery life by a factor of 3.

TABLE I: Experimental comparison against previous work

\begin{tabular}{|c|c|c|c|}
\hline Approach & Accuracy \% & Complexity & Battery life (h) \\
\hline Previous work in [8] & 95 & 47.00 & 120.64 \\
\hline Our technique & 90 & 17.22 & 394.58 \\
\hline
\end{tabular}

\section{CONCLUSIONS}

In this paper we have addressed the problem of early detection and prediction of myocardial infarction through the use of a wearable device. In order to monitor the patients on a long-term basis, we have proposed a two-level real-time event-driven classification technique that reduces the energy consumption while maintaining a high classification accuracy. The experimental evaluation of our proposed classification technique on MI data shows that this scheme reduces the energy consumption by a factor of 3 , while still maintaining the accuracy at a medically-acceptable level.

\section{ACKNOWLEDGMENT}

This work has been partially supported by the Hasler Foundation (project no. 15048), and RTD project ObeSense (no. 20NA21 143081) evaluated by the Swiss NSF and funded by Nano-Tera.ch with Swiss Confederation financing.

\section{REFERENCES}

[1] A. L. Goldberger et al., "PhysioBank, PhysioToolkit, and PhysioNet: components of a new research resource for complex physiologic signals." Circulation, vol. 101, no. 23, pp. E215-20, jun 2000.

[2] "Heart Disease Facts \& Statistics — cdc.gov." [Online]. Available: https://www.cdc.gov/heartdisease/facts.htm

[3] G. W. Reed, J. E. Rossi, and C. P. Cannon, "Acute myocardial infarction," The Lancet, vol. 389, no. 10065, pp. 197-210, jan 2017.

[4] K. Thygesen et al., "Third Universal Definition of Myocardial Infarction," Circulation, vol. 126, no. 16, 2012. [Online]. Available http://circ.ahajournals.org/content/126/16/2020

[5] A. J. Camm et al., The ESC textbook of cardiovascular medicine. Oxford University Press, 2009.

[6] C. P. Cannon et al., "Relationship of Symptom-Onset-to-Balloon Time and Door-to-Balloon Time With Mortality in Patients Undergoing Angioplasty for Acute Myocardial Infarction,” JAMA, vol. 283, no. 22, p. 2941, jun 2000.

[7] "WHO - Prevention of Recurrences of Myocardial Infarction and Stroke Study," WHO, 2013. [Online]. Available: http://www.who.int/cardiovascular $\left\{\backslash \_\right\}$diseases/priorities/ secondary $\left\{\backslash \_\right\}$prevention/country/en/index 1.html

[8] U. R. Acharya et al., "Automated detection and localization of myocardial infarction using electrocardiogram: a comparative study of different leads," Knowledge-Based Systems, vol. 99, pp. 146-156, 2016.

[9] R. Braojos, I. Beretta, G. Ansaloni, and D. Atienza "Early Classification of Pathological Heartbeats on Wireless Body Sensor Nodes," Sensors, vol. 14 , no. 12, pp. 22 532-22 551, nov 2014.

[10] C. Cortes and V. Vapnik, "Support-vector networks," Machine Learning, vol. 20, no. 3, pp. 273-297, sep 1995.

[11] M. Ribeiro, "Gaussian probability density functions: Properties and error characterization," 2004.

[12] K.-E. Årzén, “A Simple Event-Based PID Controller,” 1999.

[13] Y. Sun, K. L. Chan, and S. M. Krishnan, "ECG signal conditioning by morphological filtering," Computers in Biology and Medicine, vol. 32, no. 6, pp. 465-479, nov 2002.

[14] J. Pan and W. J. Tompkins, "A real-time QRS detection algorithm." IEEE transactions on bio-medical engineering, vol. 32, no. 3, pp. 230-6, mar 1985.

[15] Hanchuan Peng, Fuhui Long, and C. Ding, "Feature selection based on mutual information criteria of max-dependency, max-relevance, and min-redundancy," IEEE Transactions on Pattern Analysis and Machine Intelligence, vol. 27, no. 8, pp. 1226-1238, aug 2005.

[16] "INYU - The Inner You - Home." [Online]. Available: http: //www.smartcardia.com/inyu/

[17] "STM32L1 Series - STMicroelectronics." [Online]. Available: http:// www.st.com/web/en/catalog/mmc/FM141/SC1169/SS1295?sc=stm3211

[18] S. Murali, F. Rincon, and D. Atienza, "A wearable device for physical and emotional health monitoring," in 2015 Computing in Cardiology Conference (CinC). IEEE, sep 2015, pp. 121-124. 